International Journal of Engineering \& Technology, $7(4.10)(2018) 429-431$
International Journal of Engineering \& Technology
SPC
Website: www.sciencepubco.com/index.php/IJET
Research paper

\title{
Big Data Health Care System Using Distributed Wearable Sensors
}

\author{
A. Vijayalakshmi ${ }^{1 *}$, C. John Paul ${ }^{2}$ \\ ${ }^{1}$ School of Computing Sciences and Engineering, Vellore Institute of Technology, Chennai, India \\ ${ }^{2}$ Department of Computer Applications, Alpha Arts and Science College, Chennai \\ *Corresponding author E-mail: vijayalakshmi.av@vit.ac.in
}

\begin{abstract}
The modern world facilitates a luxurious life with more comfort using technology support. The latest developments not only support the human life, but it causes problems to the humans in many ways. The primary concern of Big Data is underway in Healthcare unit. Alerting before occurring of problem is evolved with the help of big data health care solutions. Different types of sensors can be integrated with big data to produce a good solution. Our system provides a mechanism for health care systems using hidden Markov model. It acts as an intelligent system. It provides appropriate support and forwarding health related data to do real time analysis for analyzing and understanding health status.
\end{abstract}

Keywords: Actigraphy; Big Data; Health care; Hidden Markov Model; Sensors; Wrist Device.

\section{Introduction}

The number of people affected with health issues are gradually increasing day by day. Different types of wearable devices also invented in recent years [1]. Wearable sensor devices are dramatically increased to 50 percent in the recent years. For time being, many wearable devices have feasibility to mankind for the purpose of merging with a user [2]. To ascertain their well-being the physical health status may give undesirable inputs to the caretakers. As a con-sequence, availability of the large data does not totally acknowledge and utilized in the health care sectors [3]. Big data health care system for real-time analysis provides services by monitoring the people's health status in the form of continuous behavioral health condition and lengthy health condition [7]. The main focus of this paper is a wrist-wearable distributed sensors node for collecting knowledge from a human body [8]. The components used in our system are mobile device for user communication and transferring the data into the server with help of internet connectivity, and a streamline server used as big data health care system as a tool (KAA streaming analysis tool) for health monitoring.

The technology used between different types of sensors and the big data analysis is server cluster. This knowledge base is used to forward the data from mobile devices to the server [10]. A Hidden Markov model (HMM) helps to share the sensor data contingency and enormously when decreases in the transmission time [4]. It helps to balance between the loads and data storage from a massive amount of system. The HMM utilizes probability likelihood dispersion or discrete likelihood values append to private perceptions [5]. The behavior recognition task, additionally elaborated standards take note from different types of causes to establish a knowledgeable estimation of the hidden state. When the hidden states are often resolved, a massive number of accuracy results are reviewed [6].

\section{Proposed System}

The proposed system enhanced the existing method for analyzing the streaming data by using KAA ingestion system as shown in Fig.1. By using KAA ingestion system we can analyze the streaming data, when data is updating for every second through wrist device. The wrist device comprises different types of health care sensors that are used to find different types of parameters like temperature, pulse rate, heart rate and actigraphy, when user doing different types of activities like jogging, running and sleeping [8].

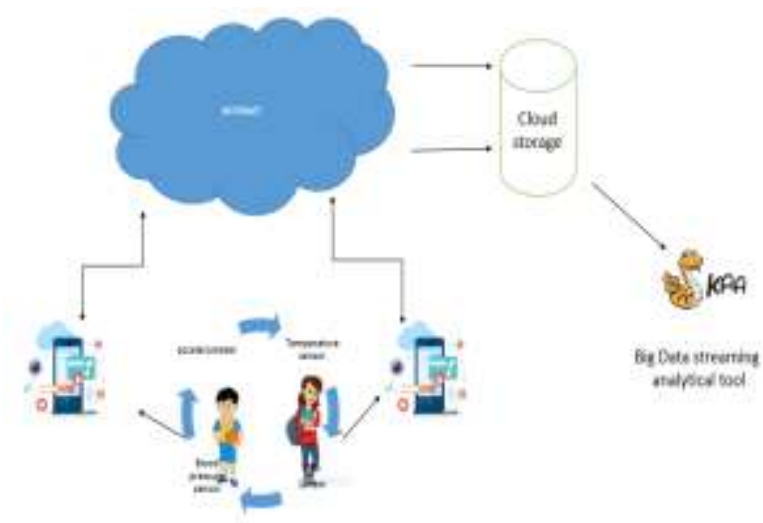

Fig. 1: KAA ingestion system

\section{Architecture}

This research exhibits big data information for Health care system. It gives people's health care information for long term by creating distributed framework comprises of three separate parts: a wrist gadget, a cell phone, and a major information group. 


\subsection{Wrist Device}

A gadget has been designed to join additional sensors and utilize Bluetooth Low Vitality (BLE) innovation for interfacing with partner android device to make a private range. It was developed by utilizing arduino board with framework on chip BLE alongside various sorts of sensors [9]. The heart rate and pulse rate are the sensors which are connecting arduino with digitally interface to read the data from different sensors.

\subsubsection{Heart Rate Sensor}

Max 30100 sensor integrated with pulse oximeter and heart-rate monitor sensor as shown in fig 2. It comprises of two LED's, a Photo detector, optimized optics, and low-noise analog signal processing for detection of pulse oximeter and heart-rate signals [10]. The led drivers IR and red led are used for pulse driver as well as spo2 and heart rate measurement. The flow of current is programmed to $0 \mathrm{~mA}$ to $50 \mathrm{~mA}$ and $200 \mathrm{us}$ to $1.6 \mathrm{~ms}$ are programmed as pulse width for optimizing power supply.

MAX30100 was developed by using software registers and the original output data was stored in 16 deep FIFO with devices. It allows connecting to microprocessor or microcontroller via a shared bus from where device registers are not continuously read the data [10]. Spo2 subsystem comprises of ambient light cancellation, time filter and 16-bit sigma delta ADC. The Spo2 algorithm was used to measure the intensive wave length of IR LED and red LED wavelength was used to correct the critical interpretation of the information. Led Driver maintains the current flows that programmed the range between $0 \mathrm{~mA}-50 \mathrm{~mA}$. Working process of led drivers as shown in fig 3 .
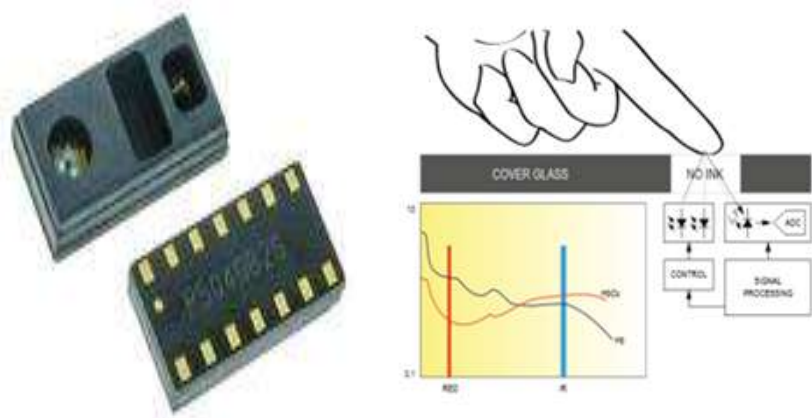

Fig. 2: Max30100 sensor

Fig. 3: Working process of led drivers

Benefits and features of Heart Rate Sensor:

Pulse Oximeter and heart-rate sensor

High performance analog front end photo sensor and integrated LED.

Tiny $5.6 \mathrm{~mm} \times 2.8 \mathrm{~mm} \times 1.2 \mathrm{~mm} 14$ pin optically enhanced system in package

Ultra-Low-Power increases battery life for wearable devices LED current for power savings and programmable sample rate. Shutdown current $(0.7 \mu \mathrm{A})$

Advanced functionality improves performance

High SNR for robust motion artifact resilience

Fast data output capability

It is integrated with ambient light cancellation

Sample rate capability is high

\subsubsection{Accelerometer}

Mc3635 belong to the family of accelerometer. It uses ultra- low power, low-noise, integrated digital output three axis accelerometer for wearable and product motion sensing. An interesting activity is recording for different types wearable devices that are conventionally producing the output data. For actigraphy uncommon word is becoming increasingly common in the sleeping field.
There are three unique philosophies that are utilized as a part of actigraphy screens to quantify physical movement.

Time above threshold.

Zero-crossing.

Digital integration

\section{Implementation and Results}

A BLE communication is used to get the parameters that are measured by the wrist device. A mobile phone having android software is used to process and collect knowledge to create a precipitate decision. The mobile device is collecting knowledge and maintains the perception of the system, as shown in Fig 4. It receives sensor observations from the wrist or finger and forward to the mobile device is shown in Fig 5.

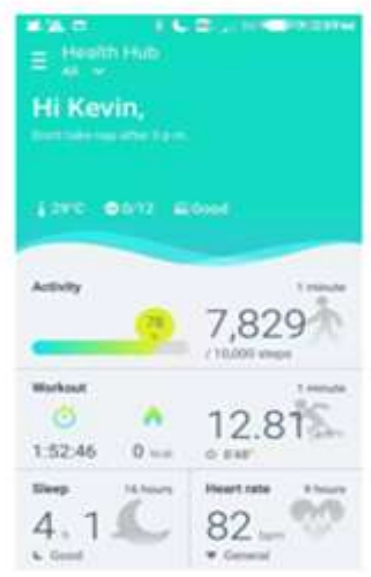

Fig. 4: Types of activities

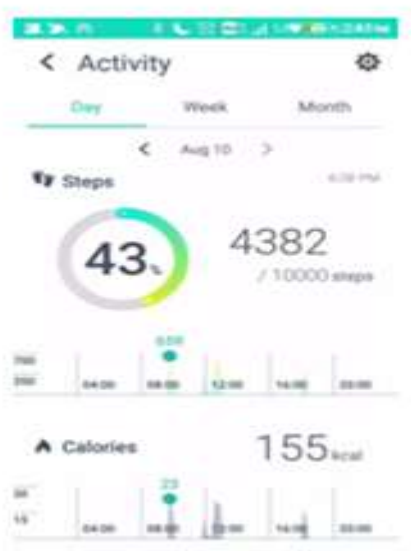

Fig. 5: Activity Schedule

The Big Data Sever is used in our system to process the enormous amount of data provided by the wearable device. The sensor rendition and the conditions of a wearer will be sent to the framework through web for analyzing. It prompts to check and customize the nature of care, affirmation the effective of rare wellbeing proficient ability. The additional potential is to achieve country patients without legitimate access of medicinal services innovation and to guarantee that patients know when and how drug ought to be balanced or utilized.

A map reduction framework is used to analyze the data which are getting from large amount of unstructured data. Map reduction acquainted by Google in 2004 to analyze huge amount of unstructured data. The traffic between the mobile device and servers are heavy. For reducing traffic we are using clusters of computers. 
These types of devices are designed to support by TCP or UDP for transferring the data between servers and mobile device.

In addition, the system is sufficiently adaptable to enter different machine-produced information streams in different formats, such as log documents, comma-isolated esteem records, databases and system administration messages through scripts. It permits the framework to associate with a substantial number of dispersed data sources with nonstandard information and eccentric. A recorder in a data stream is used to forward the information from a mobile device through internet which is written in JavaScript or python script article notation as follows.

User Name: David Carroll

Time: 09 : 20AM

Verity Battery: 90

Phone Battery: 65

Body Temp: 35.6

Heart rate: $75 \mathrm{bpm}$

Pulse rate: $60-80$

After reading the parameters from the host body, the wrist device transfers the data to the mobile device. For clearly understanding the scenario, the host has to do daily actives like running, jogging and sleeping.

\section{Conclusion}

The distributed sensor which was made in the form of wrist watch was used in big data health care analysis system to identify the patient health status. Identifying and collecting real time data from streamline servers becomes huge expenses for the company. Our system helps to analyze the data by providing a Big Data solution for their healthy living environment. In our system perceptive instruction forwarder installed along with mobile phone. Further KAA ingestion system helps to proceed with the streaming analytics.

\section{References}

[1] Meltzer LJ, Montgomery-Downs HE, Insana SP, Walsh CM, Use of Actigraphy for Assessment in Pediatric Sleep Research. Sleep medicine reviews. 2012;16(5):463-475. doi:10.1016/j.smrv.2011.10.002

[2] Sonia Ancoli-Israel, Roger Cole, Cathy Alessi, Mark Chambers, William Moorcroft, Charles P. Pollak, The Role of Actigraphy in the Study of Sleep and Circadian Rhythms, Sleep, Volume 26, Issue 3, 1 May 2003, Pages 342-392.

[3] Avi Sadeh The role and validity of actigraphy in sleep medicine: an update, Sleep machine reviews 15(2011)259-267.

[4] H.Y.K.Lau, K.L.Mak and M.C.C.Ngan, An Integrated HMM-Based Intelligent Robotic Assembly System, Springer-Verlag, 2010.

[5] Abhinav Srivastava, Amlan Kundu, Credit card Fraud Detection Using Hidden Markov Model, IEEE Transactions on dependable and secure Computing, volume: 5, issue: 1, jan-March 2008.

[6] Bhupinder Singh, Neha Kapur, Speech Recognition with Hidden Marov Model, A review: IJARST-volume 2, March 2012.

[7] D.J.Hand, G.Blunt, M.G Kelly, N.M. Adams, Data Mining for Fun and Profit, Statistical Science, vol 15, no.2, pp.111-131, 2000.

[8] Webster JB, Kripke DF, Messin S, Mullaney DJ, Wybor-ney G, An activity-based sleep monitor system for ambulatory use, Sleep. 1982; 5:389-399.

[9] Sadeh A, Sharkey KM, Carskadon MA, Activity-based sleep-wake identification: an empirical test of methodological issues, Sleep. 1994; 17:201-207.

[10] Souvik Das, The development of a micro controller based low cost heart rate counter for health care systems, IJETT-volume4 Issue 2, 2013.

[11] Nusrat Jahan Farin, S.M.A.Sharif, An intelligent sensor based system for real time heart rate monitoring (HRM), SCIRES-May 2016.

[12] Wahyu kusuma R, Ridha, FPGA Based heartbeats monitor with fingertip optical sensor", IJCSEIT,Vol. 4, No.5, oct-2014.

[13] M. Rosenblum, Generalized Hermite polynomials and the Bose-like oscillator calculus, In: Operator Theory: Advances and Applications , Birkhauser, Basel (1994), 369-396.
[14] D.S. Moak, The q-analogue of the Laguerre polynomials, J. Math. Anal. Appl. 81 (1981), 20-47. 